\title{
CONTAMINACIÓN POR METALES PESADOS DE LA MICROCUENCA AGROPECUARIA DEL RÍO HUANCARAY - PERÚ
}

Odilon Correa Cuba ${ }^{* a}$, Frida Esmeralda Fuentes Bernedo ${ }^{a}$, Rosa Gabriela Coral Surco ${ }^{\mathrm{a}}$

\section{RESUMEN}

Los metales se transportan, a nivel espacial y temporal, a través de la superficie del suelo y la atmósfera, convirtiéndose en un peligro para el medio ambiente y poblaciones vulnerables de las cuencas hidrográficas. El presente estudio tuvo como objetivo determinar fuentes y niveles de metales en sedimentos del agua en la microcuenca del río Huancaray en la región Apurímac (Perú), mediante la técnica de análisis por fluorescencia de rayos X (FRX) durante la época de secas y lluvias de los años 2018 y 2019. Los elementos detectados fueron $\mathrm{K}, \mathrm{Ca}$, Ti, V, Cr, Mn, Fe, Co, Ni, Cu, Zn, Ga, As, Br, Rb, Sr, Y, Zr, Nb y Ba. Resalta la presencia de $\mathrm{Zn}, \mathrm{Cr}, \mathrm{Cu}, \mathrm{Pb}$, Ni y As como elementos críticos en cada punto de la microcuenca del río Huancaray; las concentraciones son superiores a los límites permisibles; asimismo, los periodos de lluvia y seca no presentan diferencia significativa, mientras que las principales fuentes de contaminación son centros poblados, prácticas de la agricultura, presencia de la minería ilegal y fuente de origen natural.

Palabras clave: Andes peruanos, estiaje, fluorescencia de rayos X, precipitación, sedimentos.

\section{POLLUTION BY HEAVY METALS OF THE AGRICULTURAL MICRO-BASIN OF THE HUANCARAY RIVER - PERU}

\begin{abstract}
Metals are transported, spatially and temporally, through the ground surface and the atmosphere, generating environmental and social problems in populations located in the hydrographic basins. The present study aimed to determine sources and metal levels in water sediments in the micro-basin of the Huancaray river in Apurímac region (Peru), using the X-ray fluorescence analysis technique (XRF) during the dry and rainy season of 2018 and 2019. The elements detected were $\mathrm{K}, \mathrm{Ca}, \mathrm{Ti}, \mathrm{V}, \mathrm{Cr}, \mathrm{Mn}, \mathrm{Fe}, \mathrm{Co}, \mathrm{Ni}, \mathrm{Cu}, \mathrm{Zn}, \mathrm{Ga}, \mathrm{As}, \mathrm{Br}, \mathrm{Rb}$, $\mathrm{Sr}, \mathrm{Y}, \mathrm{Zr}, \mathrm{Nb}$ and $\mathrm{Ba}$. At each point and sampling period, critical elements have been found,

\footnotetext{
a Facultad de Ingeniería, Universidad Nacional José María Arguedas, Jr. Juan Francisco Ramos № 380, Apurímac 03000, Perú,

odiloncorrea@unajma.edu.pe, Código postal 03700
} 
as $\mathrm{Zn}, \mathrm{Cr}, \mathrm{Cu}, \mathrm{Pb}, \mathrm{Ni}$ and $\mathrm{As}$; concentrations are above permissible limits; likewise, in the rainy and dry seasons there are no significant differences, while the main sources of pollution are populated centers; agricultural practices; presence of illegal mining; and source of natural origin.

Key words: Peruvian Andes, low water, X-ray fluorescence, precipitation, sediments.

\section{INTRODUCCIÓN}

La presencia de metales pesados en agua, alimentos y aire es una de las principales problemáticas actuales a nivel mundial y en específico en el Perú. El alto grado de toxicidad de estos elementos químicos causa efectos en la salud del ser humano, pero también genera daños irreversibles en la flora y fauna, así como en el medio ambiente en general, lo cual, a su vez, produce grandes impactos socioeconómicos. En las zonas altoandinas del Perú es escasa la investigación sobre la presencia y efectos de metales, y al mismo tiempo hay una urgencia de que las cuencas y microcuencas -áreas productoras y proveedoras de productos agrícolas de primera necesidad- sean monitoreadas periódicamente. La identificación de metales pesados en aguas y sedimentos en un área tan sensible debería ser una tarea principal dentro de la gran preocupación por el control de la problemática ambiental a nivel de las cuencas y microcuencas, pues esta data permitiría la toma de decisiones políticas más acertadas a nivel de autoridades locales y regionales, e incluso del gobierno central. Es oportuno mencionar que las cuencas hídricas son receptoras de las descargas antrópicas, por lo tanto, los metales pesados tienden a depositarse en los sedimentos de los ríos, por lo que estos mismos ríos se convierten en las principales vías de trasporte de elementos químicos ${ }^{1}$.

Si bien el avance de la ciencia y tecnología ha mejorado la economía de las sociedades, también ha generado la producción de mayores y más rápidas cantidades de descargas de elementos pesados hacia las microcuencas. Hoy en día, esto constituye una amenaza a la salud de los seres vivos y es un grave problema ambiental ${ }^{2,3}$. La alta concentración de metales pesados pone en riesgo la biota por la bioacumulación y biomagnificación en órganos y tejidos en especies terrestres y acuáticas, generando un alto grado de peligrosidad y toxicidad ${ }^{4}$.

En el sector agrícola, el uso no controlado de insumos agroquímicos pone en peligro la alteración de los elementos químicos, físicos y biológicos del medio ambiente ${ }^{1}$. El desplazamiento de metales de cualquier origen a lo largo de las cuencas genera alteración sobre las propiedades físicas y químicas del agua, del suelo y del subsuelo, las que podrían, a su vez, liberarse y contaminar aguas superficiales -por acumulación biológica y cadena alimentaria-, lo que se convierte en un serio peligro inmediato para todo el país. Por lo tanto, determinar y monitorear los contaminantes en agua, fauna y flora, a través de la elaboración de mapas de peligros de contaminantes, debería ser una política activa que incluya una estrategia de mitigación ${ }^{5}$. 
Una de las características del suelo andino es la abundancia de minerales, debido a ello hay fuertes inversiones extranjeras para la extracción minera, la que normalmente se ubica en la cabecera de las cuencas hidrográficas, territorios pertenecientes a comunidades campesinas que poseen una inmensa riqueza cultural que les ha permitido convivir por siglos con esos espacios. Este cruce o convivencia forzada genera conflictos entre Estado, empresa y comunidades ${ }^{6,3}$. Vale mencionar que en algunas cuencas del Perú se determinaron altas concentraciones de metales en el intervalo entre $\mathrm{Fe}$ y $\mathrm{Be}$, a través de las técnicas de espectrometría de emisión atómica y de espectrometría de fluorescencia atómica; además, se ha encontrado que el espectrómetro de emisión óptica es la técnica más apropiada para la detección de pequeñas concentraciones de elementos químicos ${ }^{7}$.

Por otro lado, los estudios sobre la distribución de metales pesados entre ríos, desembocaduras de ríos y mares costeros, pueden ser una base sólida para desarrollar modelos predictivos sobre la distribución global de metales en los mares $^{2}$. Por ejemplo, en Tabasco (México), un estudio de metales pesados en muestras de sedimentos en la laguna de Las Ilusiones confirmó que la biota fue afectada por alta concentración de $\mathrm{Cd}, \mathrm{Ni}, \mathrm{Cr}, \mathrm{Mn}, \mathrm{Zn}, \mathrm{Pb}$ y $\mathrm{Al}^{4}$, ello significa que la determinación de metales pesados en sedimentos del agua puede ser una técnica apropiada.

Sobre el análisis por FRX, este consiste en la determinación de elementos pesados en muestras sólidas ${ }^{8}$; con esta técnica se identificaron elementos como $\mathrm{K}, \mathrm{Ca}, \mathrm{Mn}, \mathrm{Fe}, \mathrm{Cu}, \mathrm{Zn}$ y $\mathrm{Cr}$ en muestras de tabaco de cigarrillos; también se detectó en la polvareda de la ciudad de Camagüey ${ }^{9}$. Otro estudio que aplica este análisis menciona que los lagos y humedales de Caohai (China) están contaminados por metales pesados de origen natural como antropogénico ${ }^{10}$, y la presencia de $\mathrm{Hg}, \mathrm{Cd}$ y Zn está en niveles de riesgo moderado a nivel de riesgo alto. Dadas estas evidencias, se consideró que la técnica FRX era la adecuada para este estudio. Asimismo, tuvo también importancia en la decisión el ofrecimiento del Instituto Peruano de Energía Nuclear (IPEN), de Lima, para usar el equipo FRX de sus instalaciones para el análisis multielemental no destructivo y seguro. Con esta técnica ya se han realizado otros múltiples estudios en el laboratorio de FRX del IPEN, por ejemplo se destaca la eficacia de la técnica FRX a la hora de determinar la presencia de $\mathrm{Zn}, \mathrm{Cu}, \mathrm{Pb}$, entre otros, en sedimentos del río Chillón (Perú) ${ }^{13}$.

Una alta concentración de metales no siempre es atribuido a la explotación minera ${ }^{11}$, esto es algo característico de zonas andinas (presentan alta concentración de minerales). Por ejemplo, en la cuenca del Mantaro, ubicada en la sierra centro peruana, usando la técnica de espectofotometría de absorción atómica de llama ${ }^{12}$, se encontró presencia de metales pesados en las aguas de siete ríos, estos metales fueron $\mathrm{Cu}, \mathrm{Fe}, \mathrm{Pb}, \mathrm{Zn}$ y As. Las cuencas y microcuencas son las principales fuentes de producción agropecuaria y de captación del agua para consumo humano, asimismo, en estas áreas suelen establecerse ciudades y centros poblados, por lo cual es urgente cuantificar la presencia de metales. En este artículo se eligió la microcuenca del río Huancaray como área de estudio, siendo el objetivo del mismo la determinación, mediante la técnica de análisis FRX, de los niveles de concentraciones de metales en sedimentos del agua de la microcuenca del río para el periodo de estiaje y de lluvia en los años 2018 y 2019. 


\section{PARTE EXPERIMENTAL}

Se eligió como ámbito espacial de estudio a la microcuenca del río Huancaray. Como se observa en la figura 1, esta se encuentra ubicada en los Andes del sur peruano, en el departamento de Apurímac, provincia de Andahuaylas. La microcuenca involucra parte de los 10 distritos de la provincia. Se encuentra entre las coordenadas geográficas $13^{\circ} 40^{\prime}-$ $13^{\circ} 55^{\prime}$ latitud sur y $73^{\circ} 15^{\prime}-73^{\circ} 40^{\prime}$ longitud oeste, a una altitud comprendida entre los 2136 4620 m.s.n.m., con un área total de $807 \mathrm{~km}^{2}$. El principal cuerpo de agua superficial que atraviesa la microcuenca es el río del mismo nombre (Huancaray). Sus tributarios son los ríos Huancabamba, Cceñuaran, Pacchi Huaycco y Yanacollpa. A su vez, el río Huancaray es tributario del río Pampas. La microcuenca Huancaray, hidrológicamente, pertenece a la cuenca Pampas. La principal fuente de recursos hídricos en la microcuenca, al igual que en los Andes peruanos, son las precipitaciones, que alcanzan los 694,8 mm/año en la zona. El periodo de lluvias se da a partir del mes de diciembre hasta alrededor del mes de marzo, con lluvias de moderadas a escasas. En la parte alta de la microcuenca se encuentran las principales lagunas: Suytuccocha, Pucaccocha y Quilcaccocha, en esta última nace el río Huancaray.

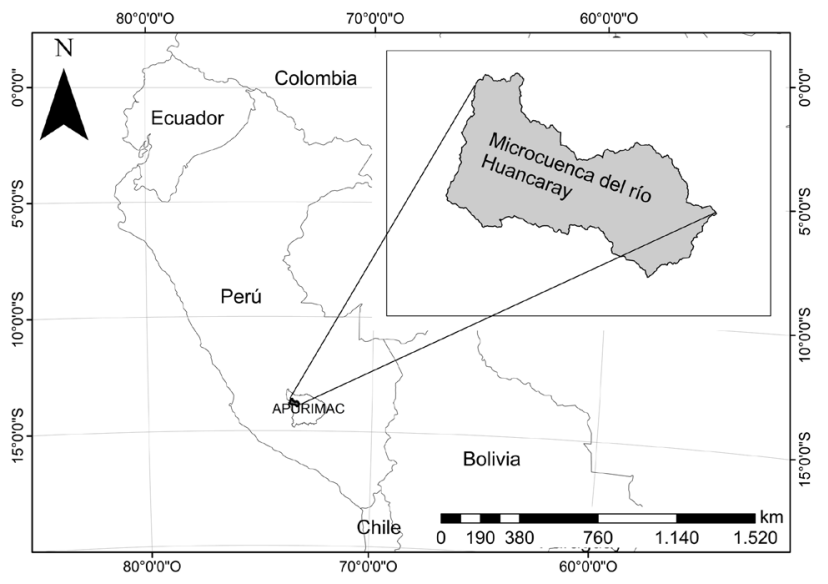

Figura 1. Ubicación geográfica de la microcuenca del río Huancaray.

Para este estudio, se ubicaron cinco puntos de muestreo por conveniencia en los lechos del río, debido a que los metales tienden a acumularse en arcillas o limo arrastrado a lo largo del río en periodos de lluvias y secas ${ }^{1}$, asimismo se consideró los factores topográficos e hidrológicos, dos puntos en la cabecera de cuenca, dos puntos en la parte intermedia y un punto en la parte baja (donde M es por "muestra" y los números del 1-5 por los cinco puntos de muestreo junto a las coordenadas UTM) sobre el río Huancaray, en la parte alta, intermedia y baja: M01 (685580-8470651) y M02 (685119-8470788) en la laguna de Suytuccocha, parte alta de la microcuenca; M03 (676249-8472311) en el centro poblado de Cceñuaran, puente 
Santa Rosa, y M04 (668817-8477418) en el centro poblado de Taypicha, estando ambos en la parte intermedia de la microcuenca; M05 (658779-8478931) en el río ubicado en la zona baja del distrito de Huancaray, parte baja de la microcuenca; la figura 2 muestra la distribución espacial de los puntos de muestreo. Los cinco puntos, M01, M02, M03, M04 y M05, se muestrearon tres veces, lo que equivale a 15 muestras. Se recogieron muestras en periodos estacionales de seca y de lluvia en las siguientes fechas: primera toma: 24 de noviembre de 2018, periodo estacional de seca; segunda toma: 19 de enero de 2019, periodo estacional de lluvia y tercera toma: 30 de marzo de 2019, periodo estacional de lluvia. En cada punto las muestras de sedimentos se recolectaron mediante una selección aleatoria simple en el lecho del río, a una profundidad entre 10 a $20 \mathrm{~cm}$, en bolsas esterilizadas Whirl Pack, a través de un colimador de cabezal en acero esmaltado AlSi 304 marca Oakf Eld Apparatus, luego se almacenaron en una caja conservadora.

Como materiales de referencia se utilizaron la Arcilla de río y Soil-7, materiales disponibles en el laboratorio de FRX del IPEN. Para la detección de metales se utilizó un equipo de medición de rayos X fluorescentes, denominada técnica FRX; para la irradiación se usó una fuente de Cd-109; para la toma de datos se empleó el detector multicanal PCAII The Nucleus, que está establecido a base de semiconductores $\mathrm{Si}(\mathrm{Li})$ de $167 \mathrm{eV}$ con resolución para una fuente de energía $5.8 \mathrm{keV}$, los límites de detección se encuentran entre $\mathrm{K}\left(\mathrm{k}_{\alpha}=3.3 \mathrm{keV}, \mathrm{k}_{\beta}=3.6\right.$ $\mathrm{keV})$ y $\mathrm{Zr}(\mathrm{k} \alpha=15.7 \mathrm{keV}, \mathrm{k} \beta=17.7 \mathrm{keV})$. La determinación cualitativa y cuantitativa se realizó con la ayuda del software QXAS.

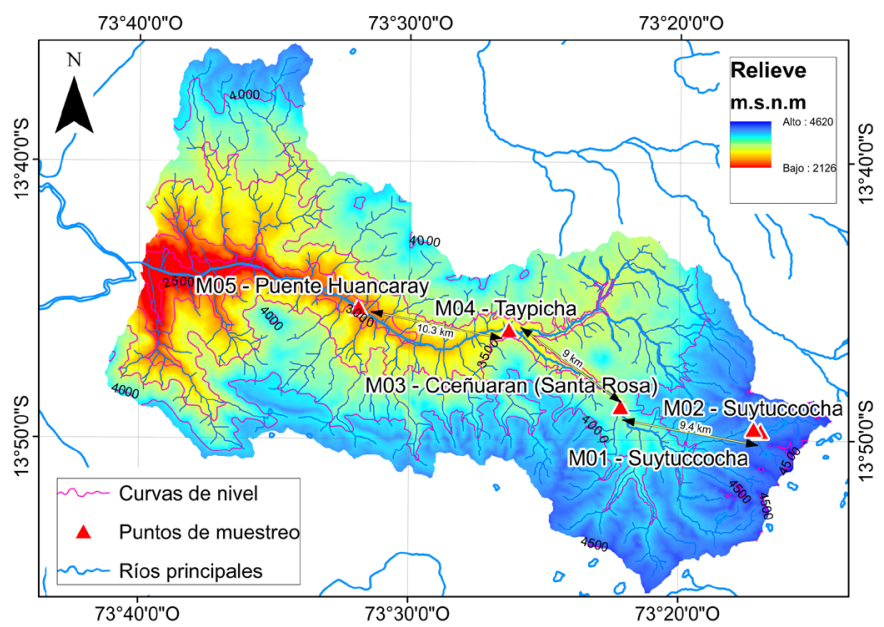

Figura 2. Puntos de muestreo, ríos principales y curvas de nivel en la microcuenca del río Huancaray. 
Las muestras se trasladaron en custodia al Laboratorio de Física de la Universidad Nacional José María Arguedas (Andahuaylas, Apurímac). Ahí, se procedió con el secado a una temperatura media de $20^{\circ} \mathrm{C}$, luego se pulverizó utilizando un mortero de ágata y finalmente fueron tamizados.

Para fines de determinación de elementos pesados, las muestras se trasladaron al laboratorio del IPEN, donde se elaboraron pastillas circulares con masas similares aproximadas a 1,7 g y un diámetro de $25 \mathrm{~mm}$. El $88 \%$ fueron muestras de sedimentos y el $12 \%$ de maicena como material aglutinante. Las variaciones en el peso y concentración del aglutinante fueron mínimas en cada caso. Para compactar muestras y lograr pastillas, se sometió a una prensa hidráulica a una presión de 30 toneladas a más, luego las pastillas se sometieron al FRX, al aire atmosférico, irradiados por un tiempo medio de $3000 \mathrm{~s}$. Haciendo uso del software QXAS, se determinó cualitativa y cuantitativamente la presencia de metales en las 15 muestras.

\section{RESULTADOS Y DISCUSIÓN}

Las 15 muestras de sedimentos tomadas en los cinco puntos de muestreo sobre el río Huancaray fueron sometidas a FRX. En las muestras M01, M02, M03, M04 y M05, tanto para el periodo de seca como de lluvia, se ha determinado al menos la presencia de 20 metales, entre metaloides, metales alcalinos, alcalinotérreos, metales de transición y otros. En la tabla 1 se pueden observar los niveles promedios de concentración, que varían para cada punto y elemento. En la figura 3 se muestra el espectro general de la presencia de elementos en sedimentos en la microcuenca estudiada. Esta es similar a la curva característica obtenida en otras cuencas hidrográficas, por ejemplo en la cuenca del Mantaro ${ }^{12}$, además es semejante al espectro característico del agua superficial encontrado en el estudio de agua de las minas de oro en Makalda ${ }^{11}$, donde se aplicó la técnica de FRX y se identificó varios elementos. La tabla 1 ilustra los límites y niveles promedios de las tres fechas de muestreo para cada punto, en total 15 muestras, donde $\mathrm{Fe}>\mathrm{Ca}>\mathrm{K}>\mathrm{Ti}>\mathrm{Mn}>\mathrm{Sr}>\mathrm{Ba}>\mathrm{Co}>\mathrm{V}>\mathrm{Cr}>\mathrm{Zr}>\mathrm{Cu}>\mathrm{Zn}>$ $\mathrm{Pb}>\mathrm{Rb}>\mathrm{Y}>\mathrm{Nb}>\mathrm{Br}>\mathrm{Ni}>$ As.

Tabla 1. Concentraciones de metales en sedimentos del río Huancaray, de Fe a Ba en $\%$ y de Co a As en $\mathrm{mg} / \mathrm{kg}(\mathrm{ppm})$.

\begin{tabular}{|c|c|c|c|c|c|c|c|c|c|c|c|c|c|c|c|c|c|c|c|c|}
\hline & $\mathrm{Fe}$ & $\mathrm{Ca}$ & K & $\mathrm{Ti}$ & $\mathrm{Mn}$ & $\mathrm{Sr}$ & $\mathrm{Ba}$ & $\mathrm{Co}$ & V & $\mathrm{Cr}$ & $\mathrm{Zr}$ & $\mathrm{Cu}$ & $\mathrm{Zn}$ & $\mathrm{Pb}$ & $\mathrm{Rb}$ & $\mathrm{Y}$ & $\mathrm{Nb}$ & $\mathrm{Br}$ & $\mathrm{Ni}$ & As \\
\hline M01 & 4,9 & 2,7 & 1,1 & 0,5 & 0,1 & 0,1 & 0,2 & 796,4 & 331,6 & 400,7 & 223,6 & 111,3 & 176,7 & 50,8 & 67,6 & 32,3 & 12,3 & 47,6 & & \\
\hline M02 & 7,9 & 4,4 & 1,4 & 0,7 & 0,1 & 0,1 & 0,3 & 1158,0 & 53,6 & 487,8 & 538,0 & 60,7 & 65,0 & 52,6 & 63,0 & 38,1 & 15,2 & 8,1 & & \\
\hline M03 & 5,1 & 5,8 & 1,1 & 0,5 & 0,1 & 0,1 & 0,3 & & 72,2 & 8 & 387,8 & & 91,1 & & 63,2 & 29,8 & 9,2 & 10,1 & & 25,3 \\
\hline M04 & 5,2 & 6,7 & 0,7 & 0,6 & 0,1 & 0,1 & 0,0 & 405 , & 554,4 & 628,8 & 122,2 & 111,4 & 104,3 & 43,3 & 37,6 & 26,1 & 5,2 & 9 & & - \\
\hline M05 & 5,8 & 10,9 & 1,6 & 0,6 & 0,1 & 0,1 & 0,0 & 479,7 & - & - & 228,2 & 92,3 & 118,7 & 62,3 & 90,6 & 23,5 & 6,5 & 17,5 & 5,4 & 50,5 \\
\hline Mín & 4,9 & 2,7 & 0,8 & 0,5 & 0,1 & 0,1 & 0,2 & 405,8 & 331,6 & 400,7 & 122,2 & 60,7 & 65,0 & 43,3 & 37,6 & 23,5 & 5,2 & 8,1 & 37,0 & 25,3 \\
\hline Máx & 7,9 & 10,9 & 1,6 & 0,7 & 0,1 & 0,1 & 0,3 & 1158,0 & 554,4 & 628,8 & 538,0 & 111,4 & 176,7 & 62,3 & 90,6 & 38,1 & 15,2 & 47,6 & 45,4 & 50,5 \\
\hline $\mathrm{DE}$ & 1,3 & 3,1 & 0,3 & 0,1 & 0,0 & 0,0 & 0,0 & 300,2 & 104,9 & 100,9 & 163,5 & 21,1 & 41,6 & 6,8 & 18,8 & 5,7 & 4,1 & 16,6 & 5,9 & 17,8 \\
\hline Med & 5,8 & 6,1 & 11,8 & 0,6 & 0,1 & 0,1 & 0,2 & 728,4 & 477,9 & 524,0 & 300,0 & 92,2 & 111,2 & 52,1 & 64,4 & 30,0 & 9,7 & 18,5 & 41,2 & 37,9 \\
\hline
\end{tabular}




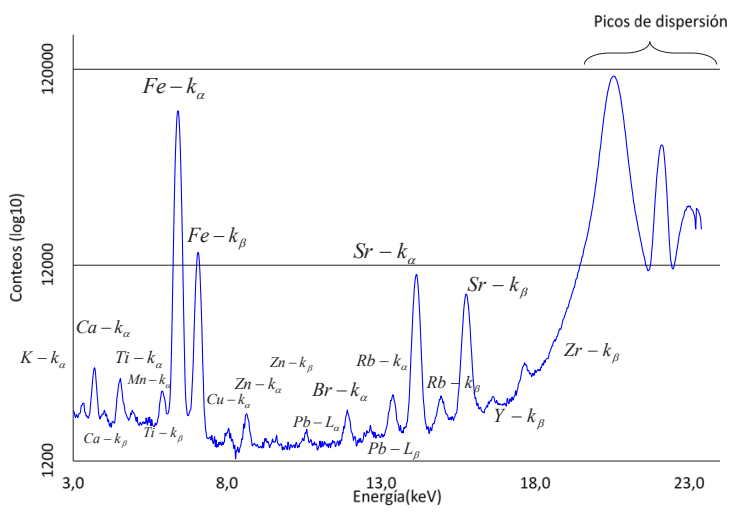

Figura 3. Espectro de una muestra de sedimentos del río Huancaray, con la técnica de Fluorescencia de Rayos X, fuente Cd-109.

Para los periodos de seca y de lluvia no se han encontrado diferencias significativas de las concentraciones de los elementos mencionados. Cuando se comparó resultados para los periodos estacionales, las variaciones tampoco fueron significativas: presentan valores medios similares y la desviación estándar es relativamente baja (tabla 1). Los puntos de muestreo se encuentran distanciados aproximadamente a $10 \mathrm{~km}$, a excepción de los puntos M01 y M02, que son muy próximos. Elementos como $\mathrm{Fe}, \mathrm{Ca}, \mathrm{K}$ y Ti son de mayor concentración para cada punto de análisis -similar situación muestra en los sedimentos de la cuenca del lago Victoria (Kenya) ${ }^{7}$, donde Fe es el elemento de mayor concentración en la naturaleza-. Ba, $\mathrm{V}$, $\mathrm{Cr}$, Ni y As son los elementos que muestran intermitencia entre los puntos de muestreo; Ba, $\mathrm{V}$ y $\mathrm{Cr}$ no tienen presencia en el último punto de muestreo, el M05.

La presencia de $\mathrm{Cr}$ se atribuye a las prácticas agropecuarias de los habitantes de la zona. Esto se explicaría porque a lo largo de la microcuenca del río de Huancaray $\left(161 \mathrm{~km}^{2}\right.$ que corresponde al $20 \%$ del total del área de la microcuenca) se practica la agricultura andina, lo cual coincide con los resultados encontrados en los sedimentos de la Bahía de Nuevitas $(\mathrm{Cuba})^{14}$, donde la presencia de $\mathrm{Cr}$ tiene origen en la industria de fertilizantes. De otro lado, Ni y As se detectaron en los puntos M04 y M05 de muestreo, la principal fuente de emisión son las aguas servidas sin tratamiento, vertidas desde los centros poblados, los cuales son cerca de 194 asentados a lo largo y ancho de la microcuenca. $\mathrm{Cr}, \mathrm{Cu}, \mathrm{Zn}, \mathrm{Pb}, \mathrm{Ni}$ y As fueron detectados en este estudio, estos elementos, según el estudio realizado en la microcuenca del río Grijalva, frontera México-Guatelama ${ }^{1}$ representan potencialidad en toxicidad. La cordillera de los Andes se extiende a lo largo del territorio peruano, siendo además cabecera de la cuenca amazónica. Se compone de variedades de climas, pisos ecológicos, zonas de vida, con presencia de recursos minerales de alta concentración. En relación a presencia minera, $65 \mathrm{~km}^{2}$, que corresponde al $8 \%$ del total de la microcuenca de Huancaray, presenta catastro minero; además, los cuerpos de agua drenan hacia el río principal, más que todo producto de las lluvias, donde la precipitación media anual es 694,8 mm/año, elemento 
climático condicionante de la sedimentación de gran parte de los minerales en las zonas altoandinas. Si se quiere usar casos de comparación de límites de presencia de químicos, no existen normas peruanas sobre los niveles estandarizados para dichas concentraciones de elementos pesados en sedimentos de agua dulce; por esa razón, para fines de análisis comparativo y discusión, se utilizaron las siguientes normas mexicana y canadiense -las que fueron recopiladas para el estudio de metales en el agua y sedimentos en la cuenca alta del río Grijalva, frontera entre México y Guantemala ${ }^{1}-$, con el objetivo de tener valores referenciales para la evaluación de impactos ambientales:

NOM: Norma Oficial Mexicana: establece criterios para determinar suelos contaminados por metales.

NOAA: Administración Oceanográfica y Atmosférica de los Estados Unidos de América.

ISQG: Guía Provisional de Calidad del Sedimento (Guías de Calidad Ambiental Canadiense).

PEL: Nivel de Efecto Probable (Guías de Calidad Ambiental Canadiense).

ECA: Aguas superficiales destinadas para recreación, DS Nº 004-2017-MINAM, Estándares de Calidad Ambiental para agua.

Por otro lado, para situaciones de discusión de resultados se utilizaron dos materiales de referencia: Soil-7 (material de referencia certificado) ${ }^{14}$ y Arcilla de río. En todos los puntos de muestreo, se detectaron $\mathrm{Fe}, \mathrm{Ca}, \mathrm{K}$ y Ti, siendo estos además de mayor concentración, algo característico de los suelos andinos. Sin embargo, altos niveles de concentración de Fe podrían constituirse en un contaminante ${ }^{14}$ cuando entra a la etapa de especiación.

En los puntos de M01 a M05 se detectó V con una concentración media de 477,9 ppm, resultado superior al parámetro de la norma NOM; además, este elemento no aparece en la arcilla de río y Soil-7. El Cr, detectado en los primeros cuatro puntos de muestreo, tiene una media de $524 \mathrm{ppm}$, comparado con las normativas NOM, NOAA, ISQG y PEL, y con los materiales de referencia arcilla de río y Soil-7, es superior. El Mn tiene una media de 1271,5 ppm, que aparentemente se encuentra dentro de los valores cuantificados en materiales de referencia y no existen valores estandarizados en otras normatividades mencionadas. El Co, en todos los puntos de muestreo, tiene una media de $728,4 \mathrm{ppm}$, que es superior a los valores que presentan los materiales de referencia arcilla de río y Soil-7, no existiendo referencia en otras normas. 
Tabla 3. Concentraciones en $\%$ y $\mathrm{mg} / \mathrm{kg}(\mathrm{ppm})$ de elementos en sedimentos de la microcuenca para los cinco puntos de muestreo, materiales de referencia y límites permisibles para metales en sedimentos establecidos por las normas NOM, NOAA, ISQG, PEL y ECA.

\begin{tabular}{cccccccc|ccccc}
\hline & & & & \multicolumn{1}{c}{ Arcilla } & \multicolumn{1}{c}{ Soil - } & & & & \\
& M01 & M02 & M03 & M04 & M05 & de río & 7 & NOM & NOAA & ISQG & PEL & ECA \\
\hline $\mathrm{K}(\%)$ & 1,1 & 1,4 & 1,1 & 0,7 & 1,6 & 1,3 & 2,0 & & & & & \\
$\mathrm{Ca}(\%)$ & 2,7 & 4,4 & 5,8 & 6,7 & 10,9 & 24,1 & 1,4 & & & & & \\
$\mathrm{Ti}(\%)$ & 0,5 & 0,7 & 0,5 & 0,6 & 0,6 & 0,3 & 0,4 & & & & & \\
$\mathrm{~V}$ & 331,6 & 553,6 & 472,2 & 554,4 & & & & 78 & & & & 0,1 \\
$\mathrm{Cr}$ & 400,7 & 487,8 & 578,8 & 628,8 & & 165,9 & & 280 & 7 a 13 & 37,3 & 90 & 0,05 \\
$\mathrm{Mn}(\%)$ & 0,1 & 0,1 & 0,1 & 0,1 & 0,1 & 0,1 & 0,1 & & & & & 0,1 \\
$\mathrm{Fe}(\%)$ & 4,9 & 7,9 & 5,1 & 5,2 & 5,8 & 3,6 & 2,6 & & & & & 0,3 \\
$\mathrm{Co}$ & 796,4 & 1158,0 & 802,1 & 405,8 & 479,7 & 293,4 & 191,9 & & & & & \\
$\mathrm{Ni}$ & & & & 37,0 & 45,4 & 68,4 & 35,6 & 1600 & 9,9 & & & 0,02 \\
$\mathrm{Cu}$ & 111,3 & 60,7 & 85,3 & 111,4 & 92,3 & 45,4 & 26,1 & & 10 a 25 & 35,7 & 197 & 2 \\
$\mathrm{Zn}$ & 176,7 & 65,0 & 91,1 & 104,3 & 118,7 & 144,4 & 87,2 & & 7 a 38 & 123 & 315 & 3 \\
$\mathrm{As}$ & & & 25,3 & & 50,5 & & & 22 & 1,1 & 5,9 & 17 & 0,01 \\
$\mathrm{Br}$ & 47,6 & 8,1 & 10,1 & 9,4 & 17,5 & 25,3 & 16,0 & & & & & \\
$\mathrm{Rb}$ & 67,6 & 63,0 & 63,2 & 37,6 & 90,6 & 131,1 & 179,9 & & & & & \\
$\mathrm{Sr}$ & 708,4 & 1111,4 & 764,2 & 1297,1 & 903,1 & 268,2 & 168,6 & & & & & \\
$\mathrm{Y}$ & 32,3 & 38,1 & 29,8 & 26,1 & 23,5 & 57,2 & 51,6 & & & & & \\
$\mathrm{Zr}$ & 223,6 & 538,0 & 387,8 & 122,2 & 228,2 & 375,1 & 410,9 & & & & & \\
$\mathrm{Nb}$ & 12,3 & 15,2 & 9,2 & 5,2 & 6,5 & 18,0 & 18,9 & & & & & 0,7 \\
$\mathrm{Ba}(\%)$ & 0,2 & 0,3 & 0,3 & & & & & 5400 & & & \\
$\mathrm{~Pb}$ & 50,8 & 52,6 & 51,6 & 43,3 & 62,3 & 174,9 & 82,3 & 400 & 4 a 17 & 35 & 91,3 & 0,01 \\
\hline
\end{tabular}

El Ni solo se ha detectado en los dos últimos puntos de muestreo (M04 y M05), con una media de 41,2 ppm, lo que lo ubica por debajo de los valores de los materiales de referencia y NOM, pero mayor a NOAA. El $\mathrm{Cu}$ hallado en los cinco puntos de muestreo tiene una concentración media de 92,2 ppm, lo que está por encima de los valores de arcilla de río y Soil-7 y por las normas NOAA, ISQG y PEL, no establecida en la NOM. El Zn presenta diferentes concentraciones, con valor medio de $92,2 \mathrm{ppm}$, lo que lo pone por debajo de los valores establecidos por ISQG y PEL, concentración similar al de los materiales de referencia; mientras para NOM es inexistente para efectos de comparación. Se ha detectado As en los puntos M03 y M05, tabla 2, que vienen a ser la parte media y la parte baja de la microcuenca, con concentraciones de $25,3 \mathrm{ppm}$ y $50,5 \mathrm{ppm}$, respectivamente, siendo estos valores superiores a las de las normas NOM, NOAA, ISQG y PEL, mientras que en los materiales de referencia arcilla de río y Soil-7 no existe. La presencia de As suele presentarse consecuencia de la extracción minera, como se ha visto en el caso del río Mantaro y proximidades ${ }^{6,12}$. En los puntos M01, M02 y M03 se encontró Ba con una concentración promedio de 2475,3 ppm, lo que está por debajo del valor establecido por NOM, en otras normas no se muestra este parámetro. Otro elemento bastante estudiado y polémico es el $\mathrm{Pb}$, los valores detectados fueron similares en los cinco puntos, tabla 2, y tiene una media de $52,1 \mathrm{ppm}$, lo que está por debajo de los valores establecidos en NOM, PEL, arcilla de río y Soil-7, pero superior a los establecidos por NOAA y ISQG. Su acumulación, en el tiempo, es muy peligrosa debido que 
en un ser vivo se distribuye en tejidos, órganos, huesos y dientes. Los elementos críticos que se hallan en concentraciones superiores a los estándares usados para este estudio, tales como Cr y As, podrían confirmar la posible contaminación de las aguas de la microcuenca del río Huancaray. Dicha presencia se atribuye a fuentes naturales y antropogénicas. En este último caso, se debe a que varios centros poblados en la zona no poseen sistemas de tratamiento de aguas residuales y las principales actividades de los pobladores, la agricultura y ganadería, generan la presencia elevada de $\mathrm{Co}, \mathrm{Cr}, \mathrm{Pb}, \mathrm{Zn}$, entre otros elementos; la presencia de los elementos $\mathrm{Fe}, \mathrm{Cr}, \mathrm{Pb}, \mathrm{Zn}$ se atribuye a fuentes antrópicas y naturales, debido a la presencia de efluentes industriales, similar caso determinado en los sedimentos del río Amarillo (China) ${ }^{15}$.

Los Andes sudamericanos son una fuente amplia de minerales, producto de ello existen las explotaciones mineras legales y artesanales o ilegales, en la parte alta de la microcuenca del río Huancaray existen varias mineras ilegales. La figura 4 muestra la manera de extracción de $\mathrm{Au}$ y $\mathrm{Cu}$, en la misma área se realiza el proceso de amalgamación para extraer $\mathrm{Au}$; se sabe que el As es producto de la explotación y procesamiento del $\mathrm{Cu}$; por lo que en este estudio atribuimos la presencia de As a las prácticas de los extractores de minerales en la parte alta de la microcuenca, la cual es además el área menos poblada. De los 20 elementos detectados, la mayoría superan las normas internacionales utilizadas en este estudio como materiales de referencia, lo que significa que la combinación de estos elementos en los sedimentos representa una peligro para la vida y ecología por ser tóxico; por ejemplo, a partir de un estudio de elementos pesados en el agua del lago y los sedimentos superficiales del humedal de la meseta de Caohai (China), se considera que si la presencia de $\mathrm{Hg}$, Cd y $\mathrm{Zn}$ se encuentra en niveles de moderado y alto en la naturaleza, la ecología está contaminada ${ }^{10}$. Así, la determinación cualitativa y cuantitativa de la presencia de metales en la microcuenca del río Huancaray es de vital importancia debido a que hay escasos estudios de esta zona; siendo cabecera de las cuencas del territorio apurimeño y tributario del río Pampas, así como principal zona de producción agrícola, con una amplia diversidad de especies de papa y maíz, el riesgo de toxicidad puede generar una alerta nacional. En tal sentido, los resultados del presente estudio serán referenciales para estudios de impactos ambientales, niveles comparativos posteriores, así como también para futuros monitoreos de las distintas cuencas del país.

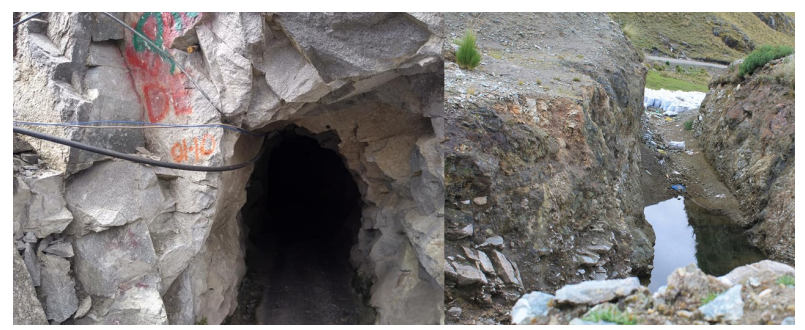

Figura 4. Presencia de minería ilegal en la parte alta de la microcuenca del río Huancaray. 
La figura 5 muestra la presencia espacial de elementos tóxicos y probables fuentes contaminantes, tales como centros poblados, áreas del territorio con prácticas agrícolas y pecuarias, estas en su mayoría están distribuidas hacia el norte de la microcuenca; mientras que hacia el sur, este y norte del territorio hay escasos centros poblados por ser zona agreste de pajonal andino, aunque también es un área de principales lagunas de agua dulce, que son fuente de recursos hídricos para el consumo y riego de la parte media y baja de la zona, pero además en esta área también se encuentran los catastros mineros, además de la minería informal. Estas características permiten que en el tiempo las aguas se filtren al suelo y subsuelo, permitiendo bofedales y manantiales permanentes en las partes baja y media, estas características geográficas ${ }^{6}$, típicas de suelos andinos, indican que las fuentes contaminantes tienen características antropogénicas y naturales.

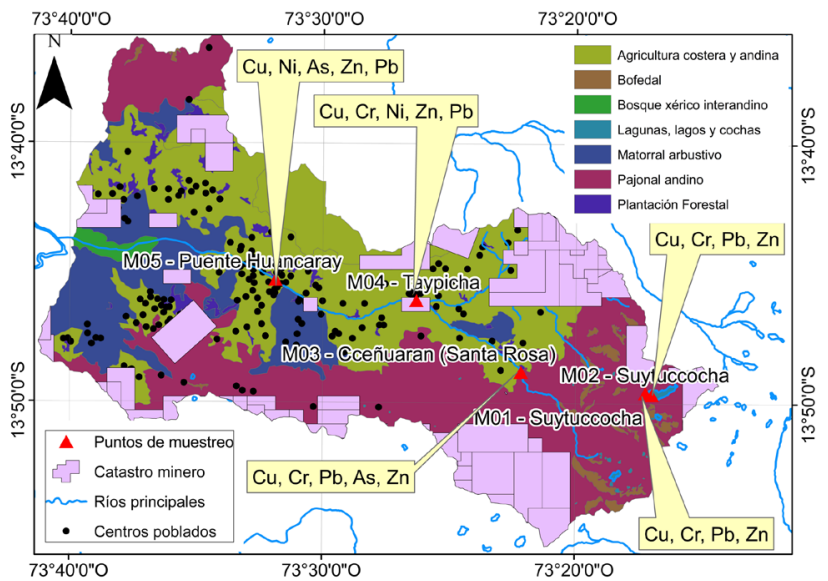

Figura 5. Metales potencialmente tóxicos, centros poblados, zonas agrícolas y catastros mineros en la microcuenca del río Huancaray.

\section{CONCLUSIONES}

El presente estudio alcanzó a estimar cualitativa y cuantitativamente la presencia de 20 elementos pesados en la microcuenca del río Huancaray, entre metaloides, metales alcalinos, alcalinotérreos y metales de transición. Las concentraciones siguen el orden de $\mathrm{Fe}>\mathrm{Ca}>$ $\mathrm{K}>\mathrm{Ti}>\mathrm{Mn}>\mathrm{Sr}>\mathrm{Ba}>\mathrm{Co}>\mathrm{V}>\mathrm{Cr}>\mathrm{Zr}>\mathrm{Cu}>\mathrm{Zn}>\mathrm{Pb}>\mathrm{Rb}>\mathrm{Y}>\mathrm{Nb}>\mathrm{Br}>\mathrm{Ni}>\mathrm{As}$; con similares características en cada punto de muestreo. Resalta la presencia de $\mathrm{Zn}, \mathrm{Cr}, \mathrm{Cu}$, $\mathrm{Pb}$, Ni y As como elementos críticos, en cada punto de la microcuenca del río Huancaray. Las principales fuentes de contaminación, por su hidrografía e hidrodinámica, son i) la agricultura, debido a su expansión y uso de fertilizantes, ii) las aguas residuales domésticas, debido a que las descargas de todos los centros poblados drenan directamente al principal río de la microcuenca, iii) y fuente natural, debido a que el área de estudio tiene potencialidad minera con catastros mineros y presencia de explotación ilegal. 
Los resultados alcanzados en este estudio pueden usarse como evidencia para plantear políticas más severas en gestión de la contaminación ambiental por elementos contaminantes, y generar modelos predictivos a nivel de las microcuencas de los Andes del sur peruano. Asimismo, los ciudadanos de esta región no parecen ser del todo conscientes del uso excesivo de los productos agroquímicos, por lo que es indispensable que las autoridades, valiéndose de estudios que validen la presencia de elementos tóxicos, tomen medidas necesarias para un uso controlado.

\section{AGRADECIMIENTO}

Nuestro agradecimiento a la Universidad Nacional José María Arguedas por el patrocinio del presente estudio. Nuestro reconocimiento al Instituto Peruano de Energía Nuclear (IPEN) y al Laboratorio de Fluorescencia de Rayos X por brindar sus servicios a través de sus especialistas Paula Ofelia Olivera y Cynthia Cáceres. Nuestra gratitud a Alexi Reynaga por su apoyo incondicional como guía de las rutas del recorrido a lo largo y ancho de la microcuenca del río Huancaray.

\section{REFERENCIAS BIBLIOGRÁFICAS}

1. Laino-Guanes RM, Bello-Mendoza R, González-Espinosa M, Ramírez-Marcial N, Jiménez-Otárola F, Musálem-Castillejos K. Concentración de metales en agua y sedimentos de la cuenca alta del río Grijalva, frontera México-Guatemala. Tecnol Cien Agua. 2015; 6(4): 61-74.

2. Cao Q, Wang H, Li Y, Zhang Y, Zheng P, Wang R, et al. The National Distribution Pattern and Factors Affecting Heavy Metals in Sediments of Water Systems in China. Soil Sediment Contam. 2018; 27(2): 79-97.

3. Julieta G, Astrid U, Gerardo D, Catalina Q, Ana Paula L. Minería y conflictos en torno al control ambiental: la experiencia de monitoreos hídricos en la Argentina, el Perú y Colombia. GRADE. Vol. 1. Lima; 2020. 83 p.

4. Flores CM, Del Angel E, Frías DM, Gómez AL. Evaluation of physicochemical parameters and heavy metals in water and surface sediment in the ilusiones Lagoon, Tabasco, Mexico. Tecnol Cien Agua. 2018; 9(2).

5. Bose SK, Chakraborty UC. Resolutions Adopted at the General Session of the VIII All India Pediatric Conference at Vellore on the 21st December, 1956. Indian J Pediatr. 1957; 24(1): 14. doi:10.1007/BF02796157

6. Quispe-Zuniga MR, Santos F, Callo-Concha D, Greve K. Impact of heavy metals on community farming activities in the central peruvian andes. Minerals. 2019; 9(10): 1417.

7. Mwamburi J. Trace metal concentration in water and sediments of satellite lakes within Lake Victoria (Kenya) basin. Lakes Reserv Res Manag. 2009; 14(3): 203-220.

8. Ulmanu M, Anger I, T EG, Mihalache M. Rapid Determination of some heavy metals in soil using an X-ray fluorescence portable instrument. 2011; 43(3): 235-241. 
9. Díaz Rizo O, Fonticiella Morell D, Arado López JO, Borrell Muñoz JL, D’Alessandro Rodríguez K, López Pino N. Spatial distribution and contamination assessment of heavy metals in urban topsoils from Las Tunas City, Cuba. Bull Environ Contam Toxicol. 2013; 91(1): 29-35.

10. Hu J, Zhou S, Wu P, Qu K. Assessment of the distribution, bioavailability and ecological risks of heavy metals in the lake water and surface sediments of the Caohai plateau wetland, China. PLoS One. 2017; 12(12): e0189295. doi: 10.1371/journal.pone.0189295

11. Odumo OB, Mustapha AO, Patel JP, Angeyo HK. Energy dispersive X-ray fluorescence analysis of mine waters from the Migori gold mining belt in Southern Nyanza, Kenya. Bull Environ Contam Toxicol. 2011; 87(3): 260-263.

12. Custodio M, Peñaloza R, Espinoza C, Peralta-Ortiz T, Ordinola-Zapata A, SánchezSuárez H, et al. Data on the concentration of heavy metals and metalloids in lotic water of the Mantaro river watershed and human risk assessment, Peru. Data Br. 2020; 30. doi: 10.1016/j.dib.2020.105493

13. Chui HB, Miramira BT, Olivera PL, Jacay JH. Determinación de metales pesados en sedimentos del río Chillón mediante la técnica de fluorescencia de rayos X. Rev Peru Química e Ing Química. 2009; 12(2): 9-14.

14. Díaz Rizo O, Gelen Rudnikas A, Lavin Pérez R, Arencibia Caraballo G, D’Alessandro Rodríguez K. XRF Analysis of sediments from Nuevitas Bay (Cuba): assessment of current heavy metal contamination. Nucleus. 2014; (55): 11-14.

15. Ren J, Shang Z, Tao L, Wang X. Multivariate analysis and heavy metals pollution evaluation in Yellow river surface sediments. Polish J. Environ Stud. 2015; 24(3): 10411048 . 